\title{
Rasgos de personalidad e inteligencia emocional en pacientes drogodependientes internos de dos centros de rehabilitación de Lima Este
}

\author{
Personality traits and emotional intelligence in internal drug dependent patients in \\ two rehabilitation centers of Lima East
}

\author{
Raúl Stifp Zela Bravo', Diana Daysi Quispe Cuno²
}

\begin{abstract}
RESUMEN
Objetivo: Determinar la relación entre los rasgos de personalidad e inteligencia emocional en pacientes drogodependientes, de 14 a 18 años, internos en dos centros de rehabilitación de Lima Este. Metodología: El tipo de estudio es cuantitativo, de alcance correlacional y de corte transversal. La muestra estuvo conformada por 153 participantes. El modelo de los rasgos de personalidad sobre el cual se trabaja es el modelo de los cinco grandes de Costa y McCrae (1992). La escala para medir los rasgos de personalidad fue el Inventario de Personalidad NEO FFI (versión abreviada), cuya adaptación peruana fue realizada por Martínez y Cassaretto (2005). Para medir los niveles de inteligencia emocional, se utilizó el Inventario de Inteligencia Emocional ICE BARON (versión abreviada), elaborado por Baron (1997) y adaptado por Ugarriza y Pajares (2011). Resultados: Se evidenció que no existe relación significativa entre ambas variables de estudio. Además, no se halló relación significativa entre inteligencia emocional con las dimensiones de extroversión, neuroticismo, apertura a la experiencia, amabilidad y responsabilidad. Conclusión: Existen otros factores que intervienen en la relación de los rasgos de personalidad con la inteligencia emocional de los participantes.
\end{abstract}

Palabras claves: Rasgos, personalidad, inteligencia, emocional, drogodependientes.

\begin{abstract}
Objective: To determine the relationship between personality traits and emotional intelligence in drug-dependent patients, from 14 to 18 years old, inmates in two rehabilitation centers in Lima East. Methodology: The type of study is quantitative, correlational in scope and cross-sectional. The sample consisted of 153 participants. The model of the personality traits on which we work is the model of the big five of Costa and McCrae (1992). The scale to measure personality traits was the NEO FFI Personality Inventory (abbreviated version), whose Peruvian adaptation was carried out by Martínez and Cassaretto (2005). To measure emotional intelligence levels, we used the Emotional Intelligence Inventory ICE BARON (abbreviated version), prepared by Baron (1997) and adapted by Ugarriza and Pajares (2011). Results: It was evidenced that there is no significant relationship between both study variables. In addition, no significant relationship was found between emotional intelligence with the dimensions of extroversion, neuroticism, openness to experience, kindness and responsibility. Conclusion: there are other factors that intervene in the relationship of personality traits with the emotional intelligence of the participants.
\end{abstract}

Keywords: Traits, personality, intelligence, emotional, drug addicts.

${ }^{1 P}$ sicólogo Clínico, Centro de Rehabilitación, Huachipa, Lima, Perú.

${ }^{2}$ Psicologa, Centro de Terapia Infantil "Anjo Gabriel”, Lima, Perú. 


\section{INTRODUCCIÓN}

El consumo de sustancias psicoactivas es un comportamiento que data desde hace miles de años. A través del tiempo el hombre ha sido propenso a consumir sustancias que modifican el funcionamiento normal del sistema nervioso con el fin de experimentar nuevas sensaciones y/o experiencias (Centro de Información y Educación para la Prevención del Abuso de Drogas [CEDRO], 2015). Sin embargo, la drogodependencia viene considerándose desde la década de los años setenta como una auténtica epidemia y como uno de los más graves problemas socio-sanitarios, debido al costo que representa en términos de vidas, comorbilidad con patologías somáticas y psiquiátricas, delitos contra la sociedad, descenso de la productividad laboral, y el incremento de conflictos familiares (Del Moral y Fernández, 2009).

Según la Oficina Nacional de las Naciones Unidas contra la Droga y el Delito (UNODC, 2016) se calcula que en el 2015 un aproximado de 250 millones de personas consumieron algún tipo de droga por lo menos una vez, aún más inquietante es que cerca de 29,5 millones de esos consumidores, es decir, el $0,6 \%$ de la población adulta mundial, padece trastornos provocados por el consumo compulsivo de las drogas, lo que significa que la afición a las drogas y su uso descontrolado es perjudicial hasta el punto de que pueden sufrir enfermedades mentales, dependencia y necesitar tratamiento.

En cuanto al panorama nacional, CEDRO (2018) menciona que el consumo de alcohol y de otras drogas ilegales se ha convertido en un problema grave que requiere una mayor atención por las autoridades, ya que dichas drogas se comercializan dentro de la población a bajos precios. Así también, en su investigación realizada dentro del país, en el año 2017, muestra que, respecto a las drogas legales, el $79.2 \%$ de la población peruana ha consumido por lo menos una vez bebidas alcohólicas y el $52.5 \%$ consumió por lo menos una vez tabaco. En cuanto a las drogas ilegales, se observa que la sustancia con mayor prevalencia es la marihuana $(8.1 \%)$, seguida por la pasta básica de cocaína $(2.0 \%)$, el clorhidrato de cocaína (1.6\%) y el éxtasis (1\%); asimismo, se evidencia que existe una mayor predominancia de consumo en varones.

Por esta razón, es necesario identificar los factores que llevan a la población peruana a buscar este tipo de sustancias y refugiarse en ellas. Frente a esta situación, Hjelle y Ziegler, (1992) y Hosseini y Anari (2011) concuerdan en que los factores que interfieren directamente para que un individuo participe en conductas de consumo de sustancias psicoactivas son: una baja autoestima, incapacidad para manejar y regular las emociones, dificultades para expresar sus sentimientos, personalidad inestable, dificultades en sus capacidades de adaptación a las presiones del medio y la falta de habilidades para la solución de problemas.

Así mismo, Pedrero, Olivar y Puerta (2007) han encontrado que los rasgos de personalidad que predominan en una población drogodependiente son los de tipo desorganizado, melancólico y ciclotímico, asimismo encontró que en la población no adicta predomina los rasgos de tipo equilibrado. De igual manera, Sánchez y Berjano (1996) demostró que los sujetos adictos son más inestables emocionalmente, toleran menos la frustración y tienden con más frecuencia a evadir todo tipo de responsabilidad. Así también Arana y Pérez (2013), en una investigación similar a las mencionadas anteriormente, demuestra que el rasgo neuroticismo podría ser un factor de riesgo en el consumo de alcohol como estrategia de afrontamiento.

Los rasgos de personalidad, como se vio en los párrafos anteriores, son uno de los factores más relevantes que influyen en la drogodependencia, y su importancia reside en su capacidad para poder explicar y predecir el comportamiento humano (Hjelle y Ziegler, 1992). Se entiende como rasgos de personalidad a la serie de tendencias básicas y características de cada individuo, las cuales lo predisponen a actuar frente a una situación determinada. (Costa y McCrae, 1997; citado por Cloninger, 2003).

Por otro lado, Hosseini y Anari (2011) manifiestan que los problemas de salud mental, en gran medida, tienen una infraestructura emocional, dicho de forma específica, los daños sociales como el suicidio, la drogodependencia, el comportamiento criminal y los trastornos mentales son resultado de una baja autoestima, incapacidad para expresar los sentimientos y la falta de habilidades de comunicación asertiva y solución de problemas. Así también, Epstein (2012) considera que las emociones desempeñan un papel muy importante al momento de desarrollar una drogodependencia, ya que, si estas surgen en un ambiente donde 
hay intentos de alejarse de un miedo, dolor o desilusión, pueden terminar refugiándose en el fondo del abuso de sustancias psicoactivas, asimismo encuentra que los individuos drogodependientes poseen bajos niveles de inteligencia emocional a diferencia de las personas no adictas. Del mismo modo Morales (2007) menciona que las personas que carecen de una adecuada regulación emocional, están más predispuestas a generar problemáticas interpersonales aumentando así la susceptibilidad a la drogodependencia.

Según lo dicho, se puede ver que, al igual que los rasgos de personalidad, la inteligencia emocional es otro de los factores más relevantes que influye en el desarrollo de las conductas drogodependientes, ya que se muestra que las personas que no tienen un manejo adecuado de sus emociones, son más propensas a refugiarse en el mundo de las drogas. (Morales, 2007; Hosseini y Anari, 2011; Epstein, 2012). Se entiende por inteligencia emocional al conjunto de habilidades personales, emocionales y sociales que influyen en la habilidad para adaptarse y enfrentar las demandas que el entorno ejerce sobre el individuo, Baron (1997).

En los intentos por relacionar ambas variables, Hosseini y Anari (2011); Nawi, Redzuan y Hamsan (2012); Kappagoda, (2013), Yusooff, Desa, Ibrahim, Kadir y Rahman (2014); concuerdan que los rasgos de personalidad, como la extroversión, amabilidad y conciencia se correlaciona positivamente con niveles altos de inteligencia emocional, por otro lado, el rasgo neuroticismo, se correlaciona con niveles bajos de inteligencia emocional. En otras palabras, los rasgos de personalidad inestables y los niveles bajos de inteligencia emocional provocan un déficit para generar actitudes y respuestas positivas, repercutiendo en una baja capacidades de adaptabilidad y afronte frente a las presiones del medio, lo que hace que sean más propensos a desarrollar una drogodependencia, incurrir en conductas delictivas, intentos de suicidios, etc.

Frente a estos alcances, y considerando la carencia de investigaciones que correlacionan rasgos de personalidad e inteligencia emocional en una población drogodependiente, la presente investigación tiene como objetivo determinar si existe una relación significativa entre las variables ya mencionadas anteriormente en pacientes drogodependientes, de 14 a 18 años, internados en dos centros de rehabilitación de Lima Este.

\section{METODOLOGÍA}

De enfoque cuantitativo y de diseño no experimental, debido a que las variables de estudio no fueron controladas ni manipuladas. Asimismo, es de corte transversal, debido al proceso de recolección de la información, puesto que se dio en un único momento, de tipo correlacional porque buscó la relación entre las variables de estudio (Hernández, Fernández y Baptista, 2005).

\section{Muestra}

Los participantes de la investigación son varones drogodependientes de nacionalidad peruana que oscilan entre las edades de 14 a 18 años y que están internados en dos centros de rehabilitación de Lima Este. Sobre la población identificada se conformó una muestra seleccionada mediante un muestreo no probabilístico; de tipo intencional, cuyo tamaño ascendió a 153 sujetos.

\section{Instrumento}

\section{Inventario de la personalidad NEO FFI}

El Inventario de la Personalidad NEO FFI fue creado por Costa y McCrae (1992) y su adaptación y validación peruana fue realizada por Martínez y Cassaretto (2011), obteniéndose una confiabilidad por encima de 0.70 en todas las escalas y se incrementó el porcentaje de varianza total explicada a 38.29. El inventario consta de 60 afirmaciones; las respuestas se dividen en 5 categorías que van desde Totalmente en desacuerdo y termina en Totalmente de acuerdo, Los resultados se obtienen a través de niveles; alto, medio y bajo. El test está agrupado en 5 dimensiones: Neuroticismo, extraversión, apertura a nuevas experiencias, amabilidad y responsabilidad; cada una de ellas están conformada por 12 ítems.

Cuestionario de inteligencia emocional (ICE Baron)

El nombre Original de la prueba es "EQYViBarOn Emotional Quotient Inventory", cuyo 
autor es Reuven Baron. Su procedencia: Toronto - Canadá y la adaptación y validación peruana fue realizada por Ugarriza y Pajares (2005), en la que se obtuvo una confiabilidad por encima de 0.80 , lo que significa que los coeficientes de confiabilidad son bastantes satisfactorios. El inventario tiene cinco dimensiones: Componente intrapersonal $(\mathrm{CIA})$, Componente interpersonal $(\mathrm{CIE})$, Componente de adaptabilidad (CAD), Componente de manejo de estrés (CME), Impresión Positiva (IP). Consta de dos versiones, la completa que posee 60 ítems, y la abrevia con 30 ítems, siendo esta ultima la que se usó para la investigación. Su calificación es usando la escala de Likert de 4 puntos en la cual los evaluados responden a cada ítem según las siguientes opciones de respuestas: "muy rara vez", "rara vez", "a menudo" y "muy a menudo". Los puntajes altos indican niveles elevados de inteligencia emocional y social. La administración del cuestionario es individual o de forma colectiva. La duración es sin límite de tiempo, pero se estima unos 10 a $15 \mathrm{~min}$ aproximadamente.

\section{Análisis de datos}

Para el análisis estadístico se utilizó el software estadístico SPSS versión 22.0 para Windows.
Una vez recolectada la información se transfirió a la matriz de datos del software estadístico para su respectivo análisis. Los resultados obtenidos han sido ordenados en tablas para su respectiva interpretación que permitió evaluar y verificar las hipótesis planteadas. Primero, se realizó la prueba de Kolmogorov-Smirnov para precisar la distribución de la muestra. Segundo, se utilizó la prueba estadística de correlación de Spearman para determinar si existe relación significativa entre las variables de estudio.

\section{RESULTADOS}

La muestra estuvo conformada por 155 participantes. Se aprecia, en la tabla 1, que el $96.7 \%$ está conformado por participantes con edad de 16 a 18 años. Así también, se observa que el $59.6 \%$ reside con ambos padres, el $22.8 \%$ reside con solo uno y el $17.6 \%$ reside con otros familiares o apoderados. Además, se evidencia que el $77.8 \%$ de los participantes proviene de la costa, el $21.6 \%$ proviene de la sierra y el $0.7 \%$ proviene de la región selva. Finalmente, se divisa que el $51 \%$ de los participantes logró culminar sus estudios académicos, por otro lado, existe un $27.5 \%$ que aún no concluye su secundaria y un $20.9 \%$ que se encuentra cursando estudios superiores.

Tabla 1

Datos sociodemográficos de los participantes.

\begin{tabular}{lll} 
Edad & N & $\%$ \\
13 a 15 años & 5 & $3.3 \%$ \\
16 a 18 años & 148 & $96.7 \%$ \\
Con quienes reside & & \\
Ambos padres & 91 & $59.6 \%$ \\
Solo con uno & 35 & $22.8 \%$ \\
Otros & 27 & $17.6 \%$ \\
Procedencia & & \\
Costa & 119 & $77.8 \%$ \\
Sierra & 33 & $21.6 \%$ \\
Selva & 1 & $0.7 \%$ \\
Grado de instrucción & & \\
Primaria & 1 & $0.7 \%$ \\
Secundaria Completa & 78 & $51 \%$ \\
Secundaria Incompleta & 42 & $27.5 \%$ \\
Superior Incompleto & 32 & $20.9 \%$ \\
\hline
\end{tabular}


En la tabla 2, se aprecia que el $86.3 \%$ de los participantes presenta niveles altos de neuroticismo, lo que significa que muestran un bajo control emocional y son tendientes a experimentar sentimientos negativos. Por otro lado, se evidencia que la mayoría de los participantes $(81.7 \%)$ presenta niveles bajos de responsabilidad, es decir que existen dificultades para planificar y ejecutar actividades. Del mismo modo, el $63.3 \%$ de los participantes presenta niveles bajos de amabilidad, lo que indica que tienen escasas habilidades empáticas y asertivas. Así también, se observa que el $51.6 \%$ de los participantes alcanza niveles bajos de extroversión, lo que significa que prefieren estar solos o en compañía de amigos íntimos. Estos hallazgos coinciden con los estudios de Martin (2014) quien realizó una investigación, en la que uno de sus objetivos fue estudiar las dimensiones de personalidad en pacientes drogodependientes internos en un centro de rehabilitación de Chile. Los resultados señalan que, a mayortiempo de internamiento, disminuyen los niveles de extraversión y aumentan los niveles de neuroticismo. Finalmente, el 51.1\% alcanzó niveles moderados de apertura a la experiencia, es decir, en algunas ocasiones buscan experimentar sensaciones nuevas. Estos resultados se asemejan a los estudios de Pedrero, Olivar y Puerta (2007); y Sánchez y Berjano (1996), quienes concluyen que los rasgos de personalidad que predominan en una población drogodependiente son los de tipo inestable, desorganizado, melancólico, impulsivo y tienden con más frecuencia a evadir todo tipo de responsabilidad.

Tabla 2

Nivel de rasgos de personalidad en los participantes.

\begin{tabular}{|c|c|c|c|c|c|c|}
\hline & \multicolumn{2}{|l|}{ Bajo } & \multicolumn{3}{|c|}{ Moderado } & \multirow{2}{*}{$\begin{array}{l}\text { Alto } \\
\%\end{array}$} \\
\hline & $n$ & $\%$ & $n$ & $\%$ & $\mathbf{N}$ & \\
\hline Extroversión & 79 & $51.6 \%$ & 61 & $39.9 \%$ & 13 & $8.5 \%$ \\
\hline Neuroticismo & 2 & $1.3 \%$ & 19 & $12.4 \%$ & 132 & $86.3 \%$ \\
\hline Amabilidad & 103 & $67.3 \%$ & 32 & $20.9 \%$ & 18 & $11.8 \%$ \\
\hline $\begin{array}{l}\text { Apertura a la } \\
\text { Experiencia }\end{array}$ & 54 & $35.3 \%$ & 78 & $51.1 \%$ & 21 & $13.7 \%$ \\
\hline Responsabilidad & 125 & $81.7 \%$ & 26 & $17.0 \%$ & 2 & $1.3 \%$ \\
\hline
\end{tabular}

En la tabla 3 se aprecia que, en la escala general, el $98.7 \%$ de los participantes obtiene niveles bajos de inteligencia emocional, lo que significa que existen dificultades para gestionar adecuadamente sus emociones. Estos resultados coinciden con los estudios de Epstein (2012) y Romero, Mayoral y Triviño (2016), quienes concluyeron que los participantes adictos presentaban cocientes bajos de inteligencia emocional. Por otro lado, haciendo un análisis de sus dimensiones, se observa que, en la escala intrapersonal, el $73.9 \%$ alcanzó niveles moderados, esto supone que presentan habilidades adecuadas para percibir sus emociones. Seguidamente, en la escala interpersonal, se aprecia que el $98.8 \%$ presenta niveles bajos en sus habilidades para percibir los sentimientos de los demás.
Así también, en la escala de adaptabilidad, se contempla que el $57.5 \%$ presenta niveles bajos en sus capacidades de resolución de problemas. Finalmente, en la escala de manejo de estrés, se evidencia que el $71.9 \%$ alcanza niveles bajos, lo que significa que existen dificultades para manejar sus estados de ánimo y controlar sus impulsos. En este sentido Mascaraque (2015) concluye que, a mayor tiempo de consumo de drogas, la regulación emocional es menor. Por otro lado, Mayer y Salovey (1997; citado por García y Giménez, 2010) refieren que la inteligencia emocional va madurando a medida que el ser humano tenga más edad. 
Tabla 3

Niveles de inteligencia emocional en los participantes.

\begin{tabular}{|c|c|c|c|c|c|c|}
\hline & \multicolumn{2}{|l|}{ Bajo } & \multicolumn{2}{|c|}{ Moderado } & \multicolumn{2}{|c|}{ Alto } \\
\hline & $n$ & $\%$ & $\mathbf{N}$ & $\%$ & $\mathbf{N}$ & $\%$ \\
\hline Escala General & 151 & $98.7 \%$ & 2 & $1.3 \%$ & 0 & $0 \%$ \\
\hline Intrapersonal & 29 & $19 \%$ & 113 & $73.9 \%$ & 11 & $7.2 \%$ \\
\hline Interpersonal & 142 & $98.8 \%$ & 11 & $7.2 \%$ & 0 & $0 \%$ \\
\hline Adaptabilidad & 88 & $57.5 \%$ & 58 & $37.9 \%$ & 7 & $4.6 \%$ \\
\hline Manejo de estrés & 110 & $71.9 \%$ & 41 & $26.8 \%$ & 2 & $1.3 \%$ \\
\hline
\end{tabular}

Con el propósito de realizar los análisis comparativos y contrastar las hipótesis planteadas, se procedido a realizar primero la prueba de bondad de ajuste para precisar si las variables presentan una distribución normal. En ese sentido la tabla 4 presenta los resultados de la prueba de bondad de ajuste de Kolmogorov-Smirnov (K-S). Como se observa en la Tabla 15 los datos correspondientes a las 2 variables en su mayoría no presentan una distribución normal dado que el coeficiente obtenido $(\mathrm{K}-\mathrm{S})$ es significativo $(\mathrm{p}<0.05)$. Por tanto, para los análisis estadísticos correspondientes se empleará estadística no paramétrica.

Tabla 4

Prueba de bondad de ajuste a la curva normal para las variables de estudio.

\begin{tabular}{llllll} 
Variables & Dimensiones & Media & D.E. & K-S & P \\
& Extroversión & 27.39 & 5.97 & .09 & .002 \\
& Neurotisismo & 22.93 & 5.78 & .07 & .029 \\
Rasgos de personalidad & Apertura a la experiencia & 26.99 & 5.20 & .11 & .000 \\
& Amabilidad & 26.58 & 6.12 & .05 & .200 \\
& Responsabilidad & 26.88 & 6.13 & .06 & .087 \\
Inteligencia Emocional & Coeficiente Emocional & 64.39 & 9.68 & .11 & .000 \\
\hline
\end{tabular}

${ }^{*} p<0.05$

Como se puede apreciar en la tabla 5, el coeficiente de correlación de Spearman indica que no existe relación significativa entre el rasgo de extroversión y los niveles de inteligencia emocional $(r=$ -,028, $p=$,734). De igual forma, se aprecia que tampoco hay relación significativa con los rasgos de neuroticismo $(r=, 086 p=, 734)$, apertura a la experiencia $(r=, 085 p=, 297)$, amabilidad $(r=, 045 p=$ ,581) y responsabilidad $(r=, 005 p=, 955)$.

Tabla 5

Coeficiente de relación entre rasgos de personalidad e inteligencia emocional.

\begin{tabular}{llc} 
Rasgos de Personalidad & \multicolumn{2}{c}{ Inteligencia emocional P } \\
rho &,- 028 &, 734 \\
Extraversión &, 086 &, 293 \\
Neuroticismo &,- 085 &, 297 \\
Apertura a la Experiencia &,- 045 &, 581 \\
Amabilidad &,- 005 &, 955 \\
Responsabilidad & \\
\hline
\end{tabular}

** La correlación es significativa al nivel 0.01 


\section{DISCUSIÓN}

En esta investigación con respecto al rasgo de personalidad neuroticismo, siguiendo el modelo de Costa y McCrae (1992) no se relaciona significativamente con la inteligencia emocional del modelo de Baron (1997), $(r=.086 p=.734)$. Esto indica que los rasgos neuróticos de los pacientes drogodependientes no se asocian con los niveles de inteligencia emocional, es decir, un participante puede tener altos o bajos niveles de neuroticismo (tendencia a la inseguridad y a experimentar sentimientos negativos) independientemente de sus niveles de inteligencia emocional. Estos resultados podrían indicar la presencia de otros factores como el funcionamiento social familiar, autoestima y las habilidades sociales, que intervienen al momento de determinar los niveles de inteligencia emocional de una persona (Goleman, 1995; Roca, 2013). Sin embargo, estos resultados difieren con los estudios de Coico y Guevara (2011); Hosseini y Anari (2011) y Nawi, Redzuan y Hamsan (2012) quienes concluyen que si existe relación significativa entre neuroticismo e inteligencia emocional. Los mismos resultados, coinciden con el estudio de Parodi, Bemonte, Ferrándis y Ruíz (2017); Alghamdi, Aslam y Khan (2017) y Arana y Caritimari (2017) quienes concluyeron que ambas variables no presentan relación significativa. Estas variaciones en los resultados, probablemente se deban a las distintas poblaciones de estudio, puesto que este puede ser un factor determinante e influyente en los resultados de una investigación.

Así también, los resultados muestran que no existe relación significativa entre el rasgo de personalidad extraversión e inteligencia emocional $(r=-.028, p=.734)$, en otros términos, los niveles de extroversión, evaluados desde la perspectiva de los pacientes, son independientes a los niveles de inteligencia emocional. Estos resultados no coinciden con los estudios de Fortes, M., Oriol, X., Filella, G., Del Arco, I. y Soldevila, A. (2013); Kappagoda (2013); Yusooff, Desa, Ibrahim, Kadir y Rahman (2014) quienes concluyeron que si existe relación significativa entre ambos constructos. Probablemente la explicación se deba a la naturaleza de esta variable, la cual de acuerdo al modelo teórico de Costa y McCrae (1992) es definida como la tendencia a ser amistosos, locuaces, despreocupados y amantes de la aventura y el riesgo. De este modo, si se hace un análisis introspectivo a los indicadores de esta dimensión, se observa que los tales encuentran una mejor asociación con otras variables de investigación distintas a la inteligencia emocional. Tal como lo indica Bastidas (2017), quien encontró relación significativa entre el rasgo de extroversión con la variable procrastinación, o sea, a mayores niveles de extroversión, mayor será la tendencia a procrastinar (retrasar una tarea pendiente). Por otro lado, los hallazgos obtenidos respecto al segundo aspecto coinciden con el estudio de Parodi, Bemonte, Ferrándis y Ruíz (2017) y Arana y Caritimari (2017) quienes encontraron que no existe una relación significativa entre la extraversión e inteligencia emocional, concluyendo este último que la extroversión no determina el nivel de inteligencia emocional de una persona y viceversa.

Tampoco se evidencia relación significativa entre el rasgo de personalidad apertura a la experiencia, del modelo de Costa y McCrae (1992), y la inteligencia emocional $(r=.085 p=.297)$. Esto da a entender que ambas variables, evaluadas desde la perspectiva de los participantes no se asocian. Estos resultados coinciden con los estudios James, Bore y Zito (2012), quienes concluyeron que la inteligencia emocional y el rasgo de apertura a la experiencia no presentan correlación significativa. Igualmente sucede con el estudio de Mesa (2015), quien concluyó que los rasgos de amabilidad y apertura a la experiencia no muestran correlación significativa con la Inteligencia emocional. Posiblemente, la explicación se deba a la naturaleza de la dimensión, la cual de acuerdo al modelo teórico de Costa y McCrae (1992) es definida como la tendencia a experimentar nuevas sensaciones que les resulten agradables y estimulantes. En este sentido, haciendo un análisis de sus indicadores, se puede observar que es una variable independendiente a la inteligencia emocional, lo cual mostraría él porque del resultado. Sin embargo, se encontró que el rasgo apertura a la experiencia se correlaciona en gran manera con la variable adicción a sustancias psicoactivas, esto es corroborado por Amirabadi et al. (2015) y Pedrero y Rojo (2008) quienes concluyen que hay niveles altos en el rasgo apertura a la experiencia que se correlacionan significativamente con el riesgo a la adicción de sustancias psicoactivas.

Del mismo modo, no se encontró relación significativa entre el rasgo de personalidad amabilidad, del modelo de Costa y McCrae 
(1992), y la inteligencia emocional $(r=.045 p$ $=.581$ ), lo que significa que ambas variables, que fueron medidas desde la perspectiva de los participantes, no se asocian. Estos resultados concuerdan con los estudios de Petrides et al. (2007) y Mesa (2015) quienes concluyeron que el rasgo de amabilidad no muestra correlación significativa con la inteligencia emocional. Probablemente esto se deba a que la variable, que es definida como la tendencia a llevarse bien con los demás, evitar los conflictos y mostrarse complaciente (Cassaretto, 2009), es independiente y no determina los niveles de inteligencia emocional, que es definida como aquellas habilidades que nos permiten gestionar adecuadamente las emociones propias y la de los demás (Baron, 1997). Sin embargo, como se menciona anteriormente, estos resultados podrían indicar la presencia de otras variables, como el funcionamiento social familiar, autoestima y las habilidades sociales, que se asocian mejor con la inteligencia emocional (Goleman, 1995; Roca, 2013). Por otro lado, existen estudios como los de Nawi, Redzuan y Hamsan (2012); Fortes, Oriol, Filella, Del Arco y Soldevila (2013); Kappagoda (2013) y Yosooff, Desa, Ibrahim, Kadir y Rahman (2014) que difieren con los resultados de este cuarto aspecto. No obstante, es menester conocer que tales resultados provienen de una población no adicta, es decir, diferente a la población del presente estudio.

Finalmente, no se halló relación significativa entre el rasgo de responsabilidad, del modelo de Costa y McCrae (1992), y la inteligencia emocional $(r=.005 p=.955)$. Estos resultados difieren con los estudios de Coico y Guevara (2011); Hosseini y Anari (2011) y Nawi, Redzuan y Hamsan (2012), quienes concluyeron que ambos constructos presentan relación significativa, vale decir, a mayores niveles de responsabilidad, mayores serán los niveles de inteligencia emocional. Sin embargo, la varianza en el resultado obtenido de la presente investigación, posiblemente se deba al tipo de población de estudio, como se mencionaba anteriormente. En este sentido, se puede afirmar que la naturaleza del rasgo de responsabilidad no se asocia con la inteligencia emocional y que existen otros factores sociales o individuales que interfieren al momento de determinar los niveles de IE en la población. Por ende, un participante puede tener altos o bajos niveles de responsabilidad (tendencia a plantearse objetivos y cumplirlos) independientemente de sus niveles de inteligencia emocional.
Declaración de financiamiento y de conflictos de interés:

El estudio fue financiado por los autores, quienes declaran no tener conflictos de interés

\section{Correspondencia:}

Raúl Stifp Zela Bravo

Correo electrónico:

raulzela@upeu.edu.pe

Diana Daysi Quispe Cuno

Correo electrónico:

diana.qc@upeu.edu.pe

\section{REFERENCIAS BIBLIOGRÁFICAS}

Alghamdi, N., Aslam, M. y Khan, K. (2017). Personality Traits as Predictor of Emotional Intelligence among the University Teachers as Advisors. Education Research International, 2017(1) 1-6. Recuperado de https://www.hindawi.com/ journals/edri/2017/9282565/abs/

Amirabadi, B., Alibeigi, N., Nikbakht, M., Massah, O., Farhoudian, A. y Doostian, Y. (2015) Rasgos de personalidad y psicopatología en nicotina y opiáceos. Revista de rehabilitación Iraní. 13(1), 56-60. Recuperado de http://irj.uswr.ac.ir/article1-485-en.pdf.

Arana, G. y Caritimari, T. (2017). Personalidad e Inteligencia Emocional en los estudiantes del nivel Primaria de la Institución Educativa Adventista José de San Martín de Tarapoto, 2016. (Tesis para optar el título de licenciado en Psicologia, Universidad Peruana Unión). Recuperado de http://repositorio.upeu.edu.pe/bitstream/handle/ UPEU/698/Gerson_Tesis_bachiller_2017. pdf? sequence $=5$ \&isAllowed $=\bar{y}$.

Bastidas, J. (2017). Procrastinación y rasgos de personalidad en estudiantes de una universidad privada de Lima este, 2016. (Tesis de licenciatura, Universidad Peruana Unión, Perú). Recuperado de http://repositorio.upeu.edu.pe/handle/ UPEU/411.

Cassaretto, M. (2009). Relación entre las cinco grandes dimensiones de la personalidad y el afrontamiento en estudiantes preuniversitarios de Lima Metropolitana. (Tesis para optar el grado de Magister en Psicologia, Universidad Nacional Mayor de San Marcos, Perú) Recuperado de http://cybertesis. unmsm.edu.pe/bitstream/handle/cybertesis/619/ 
Cassaretto bm.pdf; jessionid=68A1BA 5B52C8E088D429AF8B66E66FC3?

sequence $=1$.

Centro de Información y Educación para la Prevención del abuso de drogas (CEDRO, 2015), El problema de las drogas en el Perú. Depósito Legal en la Biblioteca Nacional del Perú No 2015-07674. Recuperado de http://www.repositorio.cedro.org. pe/bitstream/CEDRO/201/3/EI_problema_de_ las_Drogas.\%C3\%BAlti ma\%20ver.pdf.

Centro de Información y Educación para la Prevención del abuso de drogas (CEDRO, 2018), E I problema de las drogas en el Perú. Depósito Legal en la Biblioteca Nacional del Perú № 201808298. Recuperado de: http://www.repositorio. cedro.org.pe/bitstream/CEDRO/378/1/CEDRO. Problema\%20de\%20las\%20drog as.2018.pdf.

Costa, P., y McCrae, R. (1992). Revised NEO Personalyti Inventory (NEO PI-R) and NEO FiveFactor Inventory (NEO-FFI) Professional Manual. Psychological Assesment Resource, 36(3), 587-596. Recuperado de https://doi.org/10.1016/ S0191-8869(03)00118-1.

Coico, D. y Guevara, K. (2011). Rasgos de personalidad e inteligencia emocional en internos sentenciados de máxima seguridad. (Tesis para optar el título en psicología, Universidad Señor de Sipán). Recuperado de http://servicios.uss. edu.pe/handle/uss/1085.

Del Moral, M., y Fernández, P. (2009). Conceptos fundamentales en drogodependencias. Madrid. Médica Panamericana. Recuperado de http:// media.axon.es/pdf/71675.pdf.

Epstein, M. (2012). La Inteligencia Emocional en pacientes adictos. (Tesis de licenciatura en psicología, Universidad de Argentina). Recuperado de http://docplayer.es/54663064La-inteligencia-emocional-en- pacientes-adictostutor-dra-gongora-vanesa-autor-epstein-m-efecha-de-entrega.html.

Fortes, M., Oriol, X., Filella, G., Del Arco, I. y Soldevila, A. (2013). Inteligencia emocional y personalidad en las diferentes áreas de conocimiento de los estudiantes universitarios. Revista Electrónica Interuniversitaria de Formación del Profesorado, 16(1), 109-120. Recuperado de https:// www.redalyc.org/pdf/2170/217028056010. pdf?fbclid=IwAR0ZDpXUpO1MQN5McldwmcW 1V ojD_5QG5gUo5aOh-jltyBEjEDnTmPWkMVc.

García, M. y Giménez, S. (2010). La inteligencia emocional y sus principales modelos: propuesta de un modelo integrador. Espiral. Cuadernos del profesorado, 3(6), 43-52. Recuperado de https://dialnet.unirioja.es/servlet/ articulo?codigo $=3736408$
Hernández, R., Fernández, C., y Baptista, P. (2005). Metodología de la Investigación. México: McGraw-Hill Interamericana

Hjelle,, L. y Ziegler, D..(1992). Personality theories: Basic assumptions, research, and applications (3rd ed.). New York: Mcgraw-Hill Book Company. Recuperado de http://www.hrmars.com/admin/ pics/777.pdf

Hosseini, F., y Anari, A. (2011). The correlation between emotional intelligence and instable personality in substance abusers. Addict \& Health 2011, 3(3-4), 130-136. Recuperado de https://www.ncbi.nlm. nih.gov/pmc/articles/PMC3905527/pdf/AHJ-03130.pdf.

Mascaraque, A. (2015). Inteligencia emocional y consumo de sustancias ¿Cómo se relacionan? (Tesis de licenciatura en psicología, Universidad Pontificia Comillas, Madrid). Recuperado de https://repositorio.comillas.edu/rest/ bitstreams/2693/retrieve.

Martin, C. (2014). Dimensiones de personalidad en pacientes drogodependientes. (Tesis para optar el grado de licenciatura, Universidad de Aconcagua, Chile). Recuperado de http://bibliotecadigital. uda.edu.ar/objetos_digitales/448/tesis-3714dimensiones.pdf

Martínez, P. y Cassaretto, M. (2011). Validación del Inventario de los Cinco Factores NEO-FFI en español en estudiantes universitarios peruanos. Revista Mexicana de Psicología, 28(1), 6374. Recuperado de http://www.redalyc.org/ pdf/2430/243029630006.pdf.

Mesa, J. (2015). Inteligencia Emocional, Rasgos de Personalidad e Inteligencia Psicométrica en Adolescentes (Tesis doctoral en psicología, Universidad de Murcia). Recuperado de https:// www.tdx.cat/handle/10803/310420

Morales, N. (2007). La inteligencia, Modulo Inteligencia y Creatividad UNAD. Recuperado de: http://hdl.handle.net/10596/11740.

Nawi, N.; Redzuan, M. \& Hamsan, H. (2012). Inter relationship between emotional intelligence and personality trait of educator leaders. International Journal of Academic Research in Business and Social Sciences, 2(5), 223-224. Recuperado de http://www.hrmars.com/admin/pics/777.pdf.

Oficina Nacional de las Naciones Unidas contra la Droga y el Delito (UNODC, 2016) Informe mundial sobre las drogas. New York: Unodc Research. Recuperado de https://www. unodc.org/doc/wdr2016/WDR_2016_ExSum_ spanish.pdf.

Parodi, A., Belmonte, V., Ferrandis, C. y Ruiz, M. (2017). La relación entre la inteligencia emocional 
y la personalidad en estudiantes de educación secundaria. (Tesis de licenciatura en psicología, Universidad de Alicante). Recuperado de http://www.infad.eu/RevistalNFAD/OJS/index. php/IJODAEP/article/view/926/805

Pedrero, Olivar y Puerta (2007) El carácter como predictor de trastornos de la personalidad en adictos: la tipología caracterial de Cloninger y su abordaje psicosocial. Revista Española de

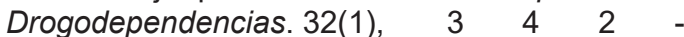
369. Recuperado de http://roderic.uv.es/ handle/10550/22358

Pedrero, E. y Rojo, G. (2008). Diferencias de personalidad entre adictos a sustancias y población general. Revista Adicciones, 20(3), 251-261. Recuperado de http://www.redalyc.org/ articulo.oa?id=289122035005.

Petrides, K., Pita, R. y Kokkinaki, F. (2007). The location of trait emotional intelligence in personality factor space. British Journal of Psychology, 98(1), 273289. Recuperado de http://citeseerx.ist.psu.edu/ viewdoc/download?doi=10.1.1.476.8331\&rep= rep1\&type=pdf

Roca, E. (2013). Inteligencia emocional y conceptos afines: autoestima sana y habilidades sociales. Congreso de inteligencia emocional y bienestar. Zaragoza. 1(1), 1-6. Recuperado de http://www. cop.es/colegiados/pv00520/pdf/IE, \%20AE, \%20 HS.\%206p.pdf
Romero, D., Mayoral, Y. y Triviño, J. (2016). Inteligencia Emocional y percepción de riesgo en consumidores de cocaína. (Tesis de licenciatura en psicología, Universidad de Castilla La Mancha). Recuperado de https://www. actaspsiquiatria.es/repositorio/18/100/ESP/18100-ESP-72-8-699330.pdf

Sánchez, E. y Bejano, E. (1996). Características de personalidad en sujetos drogodependientes. Psicothema, 8(3), 457-463. Valencia, España. Recuperado de http://www.psicothema.com/ pdf/44.pdf

Ugarriza, N. y Pajares, L. (2005). La evaluación de la inteligencia emocional a través del inventario de BarOn ICE: NA, en una muestra de niños y adolescentes. Persona, 8(1), 1158. Recuperado de http://www.redalyc.org/ pdf/1471/147112816001.pdf

Yusooff, F., Desa, A., Ibrahim, N., Kadir, N. y Murni, R. (2014). A study of the relationship between $E Q$ and personality among lecturers at a research university. Procedia-Social and Behavioral Sciences, 114(1), 351-354. Recuperado de https://www.sciencedirect.com/science/article/pii/ S1877042813053494

Recibido: 06/12/18

Aceptado: 19/03/19 